\title{
Effectiveness and Safety of Overnight Orthokeratology with Roflufocon E High-Permeability Lens Material: A 36 Weeks Follow-Up Study
}

\author{
Hui-Hsuan Huang ${ }^{1}$, Ya-Yi Chen ${ }^{1}$, Richard $\mathrm{Wu}^{1,2}$ and Wen-Pin Lin ${ }^{1,3 *}$ \\ ${ }^{1} R \& D$ Center, Brighten Optix Corporation, Taipei, Taiwan \\ ${ }^{2}$ College of Optometry, Pacific University, Forest Grove, Oregon, USA \\ ${ }^{3}$ Department of Optometry, University of Kang Ning, Taipei 11485, Taiwan
}

*Corresponding author: Wen-Pin Lin, R\&D Center, Brighten Optix Corporation; Department of Optometry, University of Kang Ning,

Taipei 11485, Taiwan

\section{ARTICLE INFO}

Received: 慧 November 13, 2020

Published: 慧 November 24, 2020

Citation: Hui-Hsuan Huang, Ya-Yi Chen, Richard Wu, Wen-Pin Lin. Effectiveness and Safety of Overnight Orthokeratology with Roflufocon E High-Permeability Lens Material: A 36 Weeks Follow-Up Study. Biomed J Sci \& Tech Res 32(1)-2020. BJSTR. MS.ID.005204.

Keywords: Myopia; Orthokeratology; Adverse Event

Abbreviations: RGP: Rigid Gas Permeable; AL: Axial Length; FDA: Food and Drug Administration; IOL: Intraocular Lens; IOP: Intraocular Pressures; ITT: Intent-ToTreat; CCLRU: Cornea and Contact Lens Research Unit

\section{ABSTRACT}

Objective: To evaluate the safety and effectiveness of overnight orthokeratology lenses with Roflufocon E, a highly gas-permeable lens material, for the temporary and reversible correction of myopia.

Methods: Myopic individuals from 9 to 45 years of age were eligible for inclusion in the study. Participants $\leq 12$ years of age were required to have myopia between $-1.00 \sim$ 4.00D and astigmatism $\leq-1.50 \mathrm{D}$, and for those 13-45 years of age myopia between -1.00 $6.00 \mathrm{D}$ and astigmatism $\leq-1.50 \mathrm{D}$. All participants were required to have normal, healthy eyes and not be receiving any ocular medications or systemic medications likely to affect the results of visual acuity.

Each participants wore the lenses for 8 to 9 hours while asleep, and they were evaluated on day 1 and weeks 1, 2, 4, 8, 12, 24, and 36. Success was defined as uncorrected visual acuity (UCVA) $\geq 0.8(0.1 \mathrm{LogMAR})$. Orthokeratology wear was discontinued after the week 36 visit. There then followed a further three follow-up visits on day 1 and weeks 1 and 4 after the final evaluation visit.

Results: A total of 85 participants (67.1\% male) with a mean age of $15.9 \pm 6.87$ years were recruited. Baseline UCVA were $0.18 \pm 0.16(0.76 \pm 0.25 \mathrm{LogMAR})$ for the $\leq-4.00 \mathrm{D}$ group and $0.06 \pm 0.07(0.95 \pm 0.11 \mathrm{LogMAR})$ for the $>-4.00 \mathrm{D}$ group. A consistent increase in UCVA was noted from day 1 to week 36 . The success rate increased with length of time. The success rates were $99.0 \%$ and $95.7 \%$ in the $<-4.00$ and $>-4.00$ groups respectively, from day1 to week 36 . No severe complications were noted.

Conclusion: The safety and efficacy study outlined here shows orthokeratology to be a safe and effective way to control myopia. Wearing orthokeratology long-term can profoundly reduce myopia, with excellent UCVA during the day, and is applicable to teenagers in controlling myopia.

\section{Introduction}

According to the statistics in 2006, myopia prevalence was $22.9 \%$ amongst the world's population. This increased to $28.3 \%$ in 2010, with an estimated increase to 34\% in 2020 and to almost half of the world's population in 2050 [1]. The highest prevalence is found in Asia [2]. Suggested reasons for this might include greater academic pressure than other countries, extended periods of near work and less outdoor activity [3]. The incidence of myopia does not just cause refractive error; it might also potentially be related to irreversible ocular disease such as retinal detachment, 
glaucoma, choroidal neovascularization, optic disc abnormalities and posterior staphyloma [4]. These pose a serious threat to vision and have led to myopia being considered one of the most important public health issues globally. Ways to control myopia so far include corrective spectacles and contact lenses, atropine and orthokeratology [5,6].

Orthokeratology lenses are specially designed Rigid Gas Permeable (RGP) contact lenses mainly worn at night to reshape the front surface of the cornea [7]. This system flattens the cornea to reduce refractive error and obviate the need for wearing spectacles for refractive correction during the daytime. Also, due to the reshaping effect which makes the cornea thinner in the center and thicker in the periphery [8-10], this creates a peripheral defocus on the retina and it is suggested that this slows down the growth in Axial Length (AL) [11-13]. Many studies have shown that orthokeratology can slow myopia progression effectively [2,10,14-16]. Orthokeratology used to control the progression of myopia does not influence accommodation [17], corneal stroma [13] or endothelial cells [18,19]. Despite other research showing that orthokeratology can decrease contrast sensitivity and increase higher order aberration $[9,20,21]$, thereby affecting vision quality, its usage is still growing [22].

Oxygen transmissibility (DK/t) is an important parameter when prescribing a contact lens. Since orthokeratology lenses are worn overnight, higher oxygen permeability rates are needed to avoid ocular hypoxia. For these lenses, oxygen permeability therefore becomes critical. Additionally, studies have shown that the effect of myopia control seems to increase with higher DK/t [23] with a concurrent decrease in the incidence of ocular complications. In a previous study, we proved that using BOSTON Equalens II material (Oprifocon A, Dk of 85) could slow myopia progression in adults and children in Taiwan effectively and safely. Therefore, this current study was to evaluate the long-term effectiveness and safety of wearing orthokeratology lenses in a material with a Dk of 125 in a group with different myopic refractive errors and a different age group. The influence on refraction after ceasing wearing should be further discuss.

\section{Materials and Methods}

\section{Participants}

The study complied with the tenets of the Declaration of Helsinki and was approved by Tri-Service General Hospital, Taiwan (Institutional Review Board N0.2-105-03-003) and Taipei Tzu Chi Hospital, Taiwan (Institutional Review Board N0.05-FS05-091). All participants or their legal guardians provided written informed consent.

Myopic individuals from 9 to 45 years of age were eligible for inclusion in the study. Participants $\leq 12$ years of age were required to have myopia between $-1.00 \sim-4.00 \mathrm{D}$ and astigmatism $\leq-1.50 \mathrm{D}$, and for those 13-45 years of age myopia between -1.00 -6.00D and astigmatism $\leq-1.50 \mathrm{D}$. All participants were required to have normal, healthy eyes defined as no evidence of active infection involving the conjunctiva, lids, or adnexa (grade 2 or less tarsal conjunctival abnormalities were acceptable); no evidence of structural abnormalities of the lids, conjunctiva, or adnexal tissue; clear cornea with no edema, staining, or opacities as observed with slit-lamp examination; no iritis; no herpes keratitis or other disease that would contraindicate lens wear or decrease the attainability of visual acuity; no use of ocular medications.

Exclusion criteria were any disease that may affect the eye or be exacerbated by wearing contact lenses; an allergy to ingredients in the study lens care solutions; pregnant, lactating, or not using a reliable contraceptive; serious systemic disease; participants in another trial within 4 weeks of entering this study; prior intraocular or corneal surgery; Schirmer's test (without topical anesthesia) results $>10 \mathrm{~mm} / 5 \mathrm{~min}$; endothelial cell count $<2000$ cells $/ \mathrm{mm} 2$; use of systemic medications that may significantly affect vision or healing within the 2 weeks prior to entering the study; prior use of rigid contact lenses or use of soft contact lenses within the 4 weeks before entering the study.

\section{Lens Design and Fitting}

The orthokeratology lens design used in this study is one previously cleared by the US Food and Drug Administration (FDA). These were custom-designed lenses to reshape the corneal curvature according to each participant's corneal data. They were manufactured in Roflufocon E material (Dk 125) by Brighten Optix Company (Taipei, Taiwan). The lenses were manufactured in two colors to make it easier for participants to differentiate left and right. Lens diameters raged from 9.8 to $11.2 \mathrm{~mm}$. Lens curvature was determined using a participant's $\mathrm{K}$ readings followed by topography. Participants were asked to wear their lenses for 8 to 9 hours every night, and to record the wearing and removal time.

\section{Evaluation and Endpoint}

Participants received a comprehensive ophthalmological examination at the screening visit (visit 1); lenses were then dispensed at the following, dispensing visit (visit 2). Participants were then seen on day 1 and weeks $1,2,4,8,12$, and 24 (visits $3-9$, respectively) for evaluation. They came in at week 36 (visit 10) for the final visit. Orthokeratology lens wear was discontinued, and the participants then had three more follow-up visits including the next day after the final visit, and then at weeks 1 and 4 (visits 11-13) to monitor VA and corneal recovery. The day 1 visit was performed in the morning, and all other visits in the afternoon. At each visit UCVA was measured with a standardized tumbling E acuity chart placed $6 \mathrm{~m}$ from the participant. Over-refraction was performed if UCVA was below 0.8(0.1LogMAR). Autorefraction and autokeratometry were performed at each visit. Moreover, corneal topography was used with topography to measure the zone affected by the lens, while AL was measured with an Intraocular Lens (IOL) 
master and Intraocular Pressures (IOP) with a tonometer. Slit-lamp biomicroscopy was performed at each visit, and the conjunctiva and cornea were evaluated for injection, edema, and neovascularization. Peripheral staining was assessed using fluorescein. The primary endpoint was the success rate of vision correction defined as UCVA $\geq 0.8(0.1 \log$ MAR) by using a Snellen chart, and over $85 \%$ of participants achieved this at their final evaluation visit. Secondary success outcomes were the lack of clinically significant ocular health issues as determined with biomicroscopy.

\section{Statistical Analyses}

Eyes which had never previously been treated with orthokeratology and met all entry criteria were considered as the Intent-To-Treat (ITT) dataset. Category variables were presented as number and percentage, while continuous variables were presented as mean and standard deviation. Data were analyzed for both eyes for each participant.

\section{Results}

\section{Study Participants}

A total of 85 participants $(67.1 \%$ male) with a mean age of $15.9 \pm 6.87$ years was recruited at the screening visit. Combining data for both right or left eyes, mean UCVA and steep corneal curvatures (Steep K) and flat corneal curvatures (Flat K) were 0.18 (0.76 LogMAR), 7.73mm, 7.95mm for the $\leq-4.00 \mathrm{D}$ group, and for the $>-4.00 \mathrm{D}$ group these were 0.06 (0.95 LogMAR), $7.76 \mathrm{~mm}, 8.00 \mathrm{~mm}$, respectively. The mean spheres of the $\leq-4.00 \mathrm{D}$ and $>-4.00 \mathrm{D}$ groups were $-2.67 \mathrm{D}$ and $-4.76 \mathrm{D}$, respectively. And the mean cylinders for the $\leq-4.00 \mathrm{D}$ and $>-4.00 \mathrm{D}$ groups were $-0.27 \mathrm{D}$ and $-0.38 \mathrm{D}$, respectively (Table 1). As a result, the ITT population consisted of 85 participants (169 eyes), one of them with only one eligible eye was still considered ITT. Five participants did not complete all 9 months of treatment due to withdrawing their consent and were excluded from PP (per protocol) population

Table 1: *Participants characteristics $(\mathrm{N}=85)$.

\begin{tabular}{|c|c|c|}
\hline \multicolumn{2}{|c|}{ Age } & $15.9 \pm 6.87$ \\
\hline \multirow{2}{*}{ Sex } & Male & $28(32.9)$ \\
\cline { 2 - 3 } & Female & $57(67.1)$ \\
\hline VA & $\leq-4.00 \mathrm{D}$ & $0.18 \pm 0.16(0.76 \pm 0.25)$ \\
\hline (Decimal(LogMAR)) & $>-4.00 \mathrm{D}$ & $0.06 \pm 0.07(0.95 \pm 0.11)$ \\
\hline \multirow{2}{*}{ Steep K (mm) } & $\leq-4.00 \mathrm{D}$ & $7.73 \pm 0.24$ \\
\cline { 2 - 3 } & $>-4.00 \mathrm{D}$ & $7.76 \pm 0.32$ \\
\hline \multirow{2}{*}{ Flat K (mm) } & $\leq-4.00 \mathrm{D}$ & $7.95 \pm 0.24$ \\
\cline { 2 - 3 } & $>-4.00 \mathrm{D}$ & $8.00 \pm 0.32$ \\
\hline \multirow{2}{*}{ Sphere over-refraction } & $\leq-4.00 \mathrm{D}$ & $-2.67 \pm 0.94$ \\
\cline { 2 - 3 } & $>-4.00 \mathrm{D}$ & $-4.76 \pm 0.68$ \\
\hline \multirow{2}{*}{ Cylinder over-refraction } & $\leq-4.00 \mathrm{D}$ & $-0.27 \pm 0.42$ \\
\cline { 2 - 3 } & $>-4.00 \mathrm{D}$ & $-0.38 \pm 0.43$ \\
\hline \multirow{2}{*}{ Axial Length(mm) } & $\leq-4.00 \mathrm{D}$ & $24.69 \pm 0.81$ \\
\cline { 2 - 3 } & $>-4.00 \mathrm{D}$ & $25.74 \pm 0.87$ \\
\hline \multirow{2}{*}{$*$} & & \\
\hline \multirow{2}{*}{$*$} & & \\
\hline
\end{tabular}

\begin{tabular}{|l|l|l|}
\hline \multirow{2}{*}{$\mathrm{IOP}(\mathrm{mmHg})$} & $\leq-4.00 \mathrm{D}$ & $16.51 \pm 2.02$ \\
\cline { 2 - 3 } & $>-4.00 \mathrm{D}$ & $15.53 \pm 2.34$ \\
\hline
\end{tabular}

Note: *Data presented as mean \pm standard deviation, except for sex which is presented as number (percentage).

\section{Vision Correction Success Rate}

The vision correction success rate UCVA $\geq 0.8(0.1 \log M A R)$ at each of the follow-up visits is shown in Figure 1. The success rate increased with length of time after beginning treatment (from $44.4 \%$ to $99.0 \%$ in the $\leq-4.00 \mathrm{D}$ group, and from $12.9 \%$ to $95.7 \%$ in the $>-4.00 \mathrm{D}$ group, from day 1 to week 36 ). Specifically, the success rates were lower than $80 \%$ before week 2 , and reached beyond $85 \%$ at week 8 for both groups. In addition, the different refraction power on baseline did not affect the correction results.

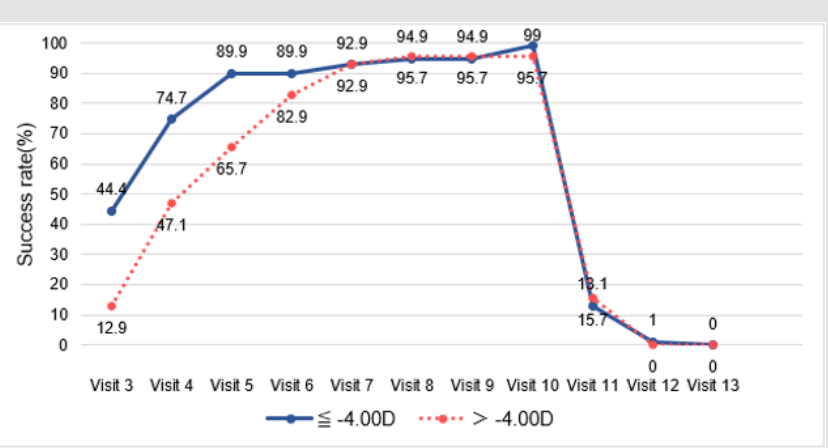

Figure 1: Treatment success rate.

Time Trends of LogMAR, Corneal Curvature, Sphere, Cylinder, Axial Length and IOP

The mean LogMAR at baseline was higher than at any of the follow-up visits. A significant decrease in LogMAR was noted from day 1 to week 36 (p-value for time trend $<0.001$ for both groups); there was a sharp decrease from day 1 to week 1 , after which LogMAR remained stable (Figure 2.1). A slight flattening in both Steep $\mathrm{K}$ and Flat $\mathrm{K}$ were noted from screening to week 1 (Figures 2.2 \& 2.3). Significant improvement of sphere was found in both groups ( $\mathrm{p}$-value for time trend $<0.001$ for both groups), and sphere reached a plateau at week 12 (Figure 2.4). All astigmatism, no matter in what refraction, were improved and back to the refraction smiliar to baseline after stop wearing lenses (Figure 2.5). The axial length did not change much and was meaningless clinically whether during the research or after stopping lens wear (Figure 2.6). In addition, IOP dropped between baseline and week 36. This might be due to thinning of the central cornea caused by orthokeratology (Table 2).

Table 2: Trends of IOP at Base line(V1) and Final visit(V10).

\begin{tabular}{|c|c|c|}
\hline & $\leq-4.00 D$ & $>-4.00 D$ \\
\hline \multicolumn{3}{|c|}{ Baseline (Visit 1) } \\
\hline $\mathrm{N}$ & 99 & 70 \\
\hline Mean \pm standard & $16.51 \pm 2.02$ & $15.53 \pm 2.34$ \\
\hline \multicolumn{3}{|c|}{ Final Visit (Visit 10) } \\
\hline
\end{tabular}




\begin{tabular}{|c|c|c|}
\hline $\mathrm{N}$ & 94 & 67 \\
\hline Mean \pm standard & $15.40 \pm 2.19$ & $14.72 \pm 2.49$ \\
\hline
\end{tabular}

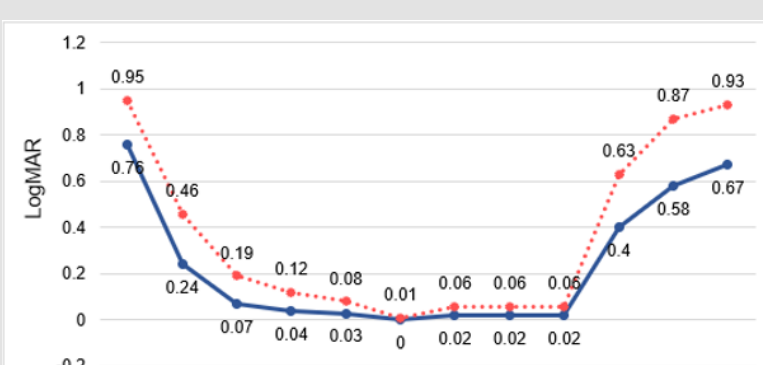

$-0.2 \quad$ Visit 1 Visit 3 Visit 4 Visit 5 Visit 6 Visit 7 Visit 8 Visit 9 Visit 10 Visit 11 Visit 12 Visit 13

$$
\longrightarrow \leqq-4.00 \mathrm{D} \cdots \cdots>-4.00 \mathrm{D}
$$

Figure 2(1): Time trends of LogMAR.

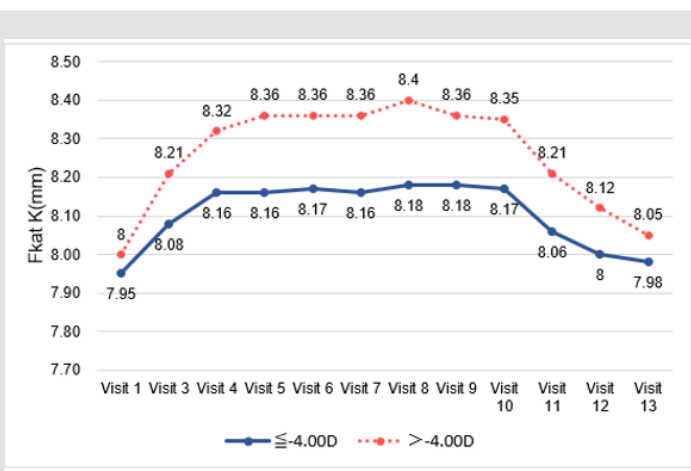

Figure 2(2): Time trends of corneal curvature (Flat K).

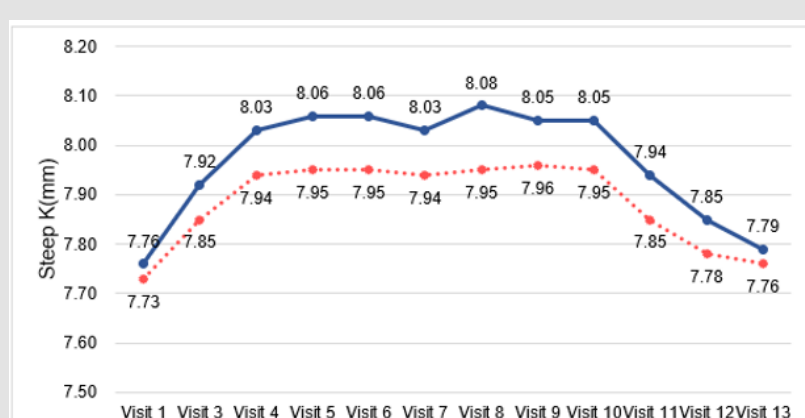

$$
\rightarrow \leqq-4.00 \mathrm{D} \quad \cdots \rightarrow \cdots>-4.00 \mathrm{D}
$$

Figure 2(3): Time trends of corneal curvature (Steep K).

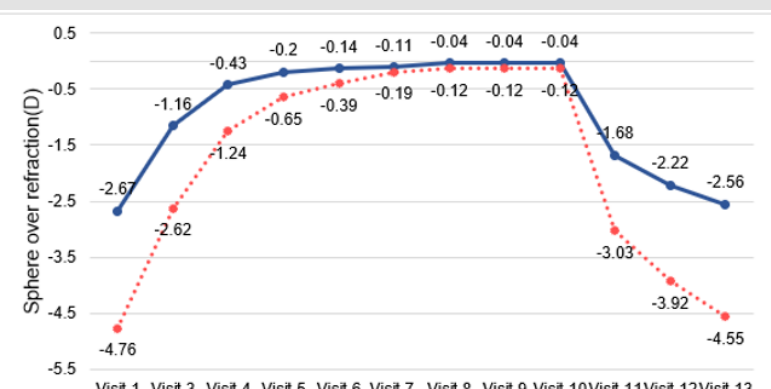

$$
\rightarrow \leqq-4.00 \mathrm{D} \quad \cdots . .>-4.00 \mathrm{D}
$$

Figure 2(4): Time trends of sphere over refraction.

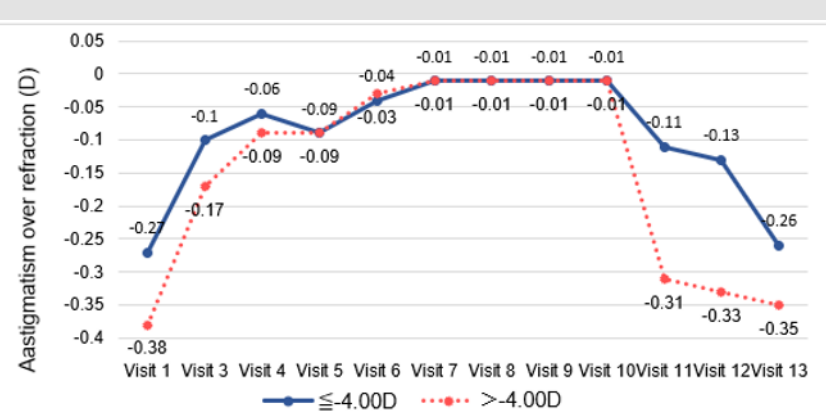

Figure 2(5): Time trends of astigmatism over refraction.

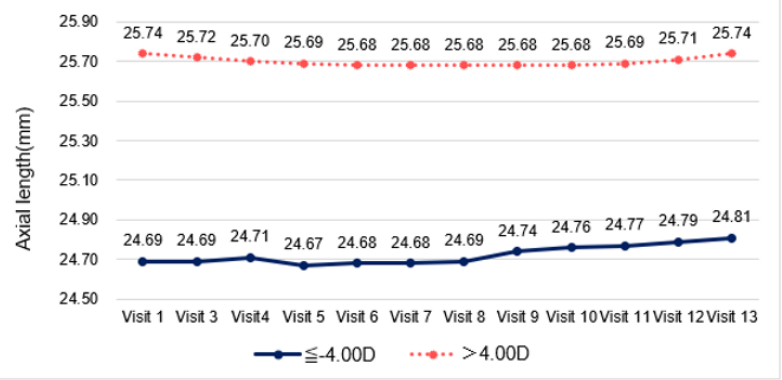

Figure 2(6): Time trends of Axial Length.

\section{Safety Evaluation}

Grading of injection, edema, neovascularization, staining, conjunctival hyperemia, palpebral conjunctival observations, other complications and dry eye classification with fluorescein was based on the Cornea and Contact Lens Research Unit (CCLRU) grading scale. No injection, edema or neovascularization was found during any visit. In 87 eyes corneal staining was noted (very slight, 80 eyes; slight, 7 eyes); in 36 eyes conjunctival hyperemia was noted (very slight, 31 eyes; slight, 5 eyes); in 24 eyes palpebral conjunctival observations were noted (very slight, 16 eyes; slight, 8 eyes); in 15 eyes other complication were noted (very slight) and dry eye was noted in 4 eyes (classification slight) (Figure 3).

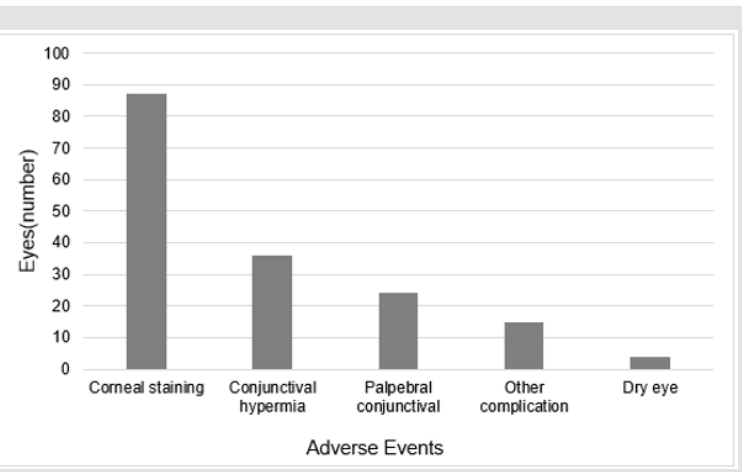

Figure 3: Safety evaluation.

\section{Discussion}

There have been several studies showing that orthokeratology can slow myopia progression effectively. In this study, corneal 
curvature increased after 36 weeks, most notably in the 1st week, both in Flat $\mathrm{K}$ and Steep K. Other studies had similar outcomes whether in radius [24] or in diopter [25,26] value. Corneal toricity is a matter of concern when fitting orthokeratology. Chen et al. [26] found increased corneal toricity after discontinuing wear of long-term orthokeratology lenses. This could be one of the major check points when assessing participants who have been long term wearers of orthokeratology lenses. An incidence of $29.6 \%$ corneal staining and other mild complications was found in this study, which is consistent with other research $[27,28]$. Infection is of course a serious issue in contact lens wear. Recent studies have shown the incidence of infection in orthokeratology to be very low $[27,29]$ which appears to be the same in this study. In addition, the effects of corneal flattening or steepening along with changes in corneal thickness were also observed. Several studies have shown that corneal thickness changes significantly after orthokeratology, especially mainly due to epithelial thinning while stromal changes had no influence or little contribution [13,30,31]. However, some studies have indicated no significant change in corneal thickness $[18,32]$.

The exact mechanisms still require long term observation in the future. Oxygen permeability is an important parameter in contact lens material. Early studies showed that the lowest permeability rate to prevent corneal swelling when wearing lenses at night was a DK/t of 87 [33]. But studies in recent years have shown that this value in a closed eye situation has to be higher than $125[34,35]$ to provide better corrective effect and lower cornea swelling [36]. This trial had some limits. Firstly, product safety is easily influenced by personal hygiene or storage conditions. Therefore, the cleanliness of the lens cannot be controlled. Secondly, corneal flexibility cannot be measured, which will cause the correction results to vary between participants. Thirdly, this study does not have a long-term followup. A longer follow-up would allow us to measure and analyze the change in all data recorded in this study. In summary, reviewing all of the research data presented in this study, this clearly shows that orthokeratology using Roflufocon E high-permeability material is a safe and effective method to temporarily and reversibly correct myopia. In this way, one can have great UCVA during the day and effectively, and reversibly, correct myopia in teenagers.

\section{References}

1. Holden BA, Fricke TR, Wilson DA, Jong M, Naidoo KS, et al. (2016) Global Prevalence of Myopia and High Myopia and Temporal Trends from 2000 through 2050. Ophthalmology 123(5): 1036-1042.

2. Wolffsohn JS, Calossi A, Cho P, Gifford K, Jones L, et al. (2019) Global trends in myopia management attitudes and strategies in clinical practice - 2019 Update. Contact Lens and Anterior Eye 43(1): 9-17.

3. Dolgin E (2015) The myopia boom. Nature 519(7543): 276-278.

4. Ikuno Y (2017) Overview Of The Complications Of High Myopia. Retina 37(12): 2347-2351.

5. Aller TA (2014) Clinical management of progressive myopia. Eye (Lond) 28(2): 147-153.
6. Chassine T, Villian M, Hamel CP, Daien V (2015) How can we prevent myopia progression? Eur J Ophthalmol 25(4): 280-285.

7. Wildsoet CF, Chia A, Cho P, Guggenheim JA, Polling JR, et al. (2019) IMI - Interventions for Controlling Myopia Onset and Progression Report. Investigative Ophthalmology \& Visual Science 60(3): M106-M131.

8. Nti AN, Berntsen DA (2020) Optical changes and visual performance with orthokeratology. Clinical and Experimental Optometry 103(1): 4454.

9. Lian Y, Shen M, Huang S, Yuan Y, Wang Y, et al. (2014) Corneal reshaping and wavefront aberrations during overnight orthokeratology. Eye Contact Lens 40(3): 161-168.

10. Swarbrick HA, Alharbi A, Watt K, Lum E, Kang P (2015) Myopia control during orthokeratology lens wear in children using a novel study design. Ophthalmology 122(3): 620-630.

11. Santodomingo-Rubido J, Villa-Collar C, Gilmartin B, Gutiérrez-Ortega R, Sugimoto K (2017) Long-term Efficacy of Orthokeratology Contact Lens Wear in Controlling the Progression of Childhood Myopia. Curr Eye Res 42(5): 713-720.

12. Tarutta EP, Verzhanskaya TY (2017) [Stabilizing effect of orthokeratology lenses (ten-year follow-up results)]. Vestn Oftalmol 133(1): 49-54.

13. Kim WK, Kim BJ, Ryu IH, Kim JK, Kim SW (2018) Corneal epithelial and stromal thickness changes in myopic orthokeratology and their relationship with refractive change. PLoS One 13(9): e0203652.

14. Sun Y, Xu F, Zhang T, Liu M, Wang D, et al. (2015) Orthokeratology to control myopia progression: a meta-analysis. PLoS One 10(4): e0124535.

15. Zhu MJ, Feng HY, He XG, Zou HD, Zhu JF (2014) The control effect of orthokeratology on axial length elongation in Chinese children with myopia. BMC Ophthalmol 14: 141.

16. Cho P, Cheung SW (2012) Retardation of Myopia in Orthokeratology (ROMIO) Study: A 2-Year Randomized Clinical Trial. Investigative Ophthalmology \& Visual Science 53(11): 7077-7085.

17. Felipe-Marquez G, Nombela-Palomo M, Cacho I, Nieto-Bona A (2015) Accommodative changes produced in response to overnight orthokeratology. Graefes Arch Clin Exp Ophthalmol 253(4): 619-626.

18. Xie PY, Chi H, Zhang Y, Zhu GM, Liu J (2007) [Effects of wearing long-term Ortho-K contact lens on corneal thickness and corneal endothelium]. Zhonghua Yan Ke Za Zhi 43(8): 680-683.

19. Yuan S, Zhang S, Jiang Y, Li L (2019) Effect of short-term orthokeratology lens or ordinary frame glasses wear on corneal thickness, corneal endothelial cells and vision correction in adolescents with low to moderate myopia. BMC Ophthalmol 19(1): 242.

20.Sanz, E, Cerviño A, Gonzalez-Meijome J (2016) Corneal Aberrations, Contrast Sensitivity, and Light Distortion in Orthokeratology Patients: 1-Year Results. Journal of Ophthalmology 2016: 1-8.

21. Santodomingo-Rubido J, Villa-Collar C, Gilmartin B, Gutiérrez-Ortega R, Suzaki A (2017) Short- and Long-Term Changes in Corneal Aberrations and Axial Length Induced by Orthokeratology in Children Are Not Correlated. Eye Contact Lens 43(6): 358-363.

22. Morgan PB, Efron N, Woods CA, Santodomingo-Rubido J (2019) International survey of orthokeratology contact lens fitting. Cont Lens Anterior Eye 42(4): 450-454.

23. Lum E, Swarbrick HA (2011) Lens Dk/t influences the clinical response in overnight orthokeratology. Optom Vis Sci 88(4): 469-475.

24. Chou CC, Huang YC, Tsai YY, Lin JM, Chen WL, et al. (2013) Changes in corneal curvature after wearing the orthokeratology lens. Taiwan Journal of Ophthalmology 3(4): 156-159.

25. Maseedupally V, Gifford P, Lum E, Swarbrick H (2013) Central and paracentral corneal curvature changes during orthokeratology. Optom Vis Sci 90(11): 1249-1258. 
26. Chen Z, Zhou J, Xue F, Zhou XT, Qu X (2018) Increased Corneal Toricity after Long-Term Orthokeratology Lens Wear. J Ophthalmol 2018 7106028.

27. Liu YM, Xie P (2016) The Safety of Orthokeratology--A Systematic Review. Eye Contact Lens 42(1): 35-42.

28. Miao CX, Xu XY, Zhang H (2017) [Analysis of corneal complications in children wearing orthokeratology lenses at night]. Zhonghua Yan Ke Za Zhi 53(3): 198-202.

29. Bullimore MA, Sinnott LT, Jones-Jordan LA (2013) The risk of microbial keratitis with overnight corneal reshaping lenses. Optom Vis Sci 90(9): 937-944.

30. Reinstein DZ, Gobbe M, Archer TJ, Couch D, Bloom B (2009) Epithelial, stromal, and corneal pachymetry changes during orthokeratology. Optom Vis Sci 86(8): E1006-E1014.

31. Wan K, Lau J KK, Cheung SW, Cho P (2019) Refractive and corneal responses of young myopic children to short-term orthokeratology treatment with different compression factors. Contact Lens and Anterior Eye 43(1): 65-72.

ISSN: 2574-1241

DOI: 10.26717/BJSTR.2020.32.005204

Wen-Pin Lin. Biomed J Sci \& Tech Res

(C) This work is licensed under Creative

Submission Link: https://biomedres.us/submit-manuscript.php
32.Guo, X, Xie P (2014) [Corneal thickness and endothelial observation for youth myopia patients fitted with ortho-k lens for seven years] Zhonghua Yan Ke Za Zhi 50(1): 9-13.

33. Holden BA, Mertz GW, Mc Nally JJ (1983) Corneal swelling response to contact lenses worn under extended wear conditions. Invest Ophthalmol Vis Sci 24(2): 218-226.

34. Moezzi AM, Fonn D, Varikooty J, Simpson T (2015) Overnight corneal swelling with high and low powered silicone hydrogel lenses. Journal of optometry 8(1): 19-26.

35. Harvitt DM, Bonanno JA (1999) Re-evaluation of the oxygen diffusion model for predicting minimum contact lens $\mathrm{Dk} / \mathrm{t}$ values needed to avoid corneal anoxia. Optom Vis Sci 76(10): 712-729.

36. Kakita T, Hiraoka T, Oshika T (2011) Influence of overnight orthokeratology on axial elongation in childhood myopia. Invest Ophthalmol Vis Sci 52(5): 2170-2174.

$\begin{array}{ll}\text { BIOMEDICAL } & \text { Assets of Publishing with us } \\ \text { RESEARCHES } & \text { - Global archiving of articles } \\ & \text { - Immediate, unrestricted online access } \\ & \text { - Rigorous Peer Review Process } \\ & \end{array}$

